\title{
Efficient Network Tomography for Internet Topology Discovery
}

\author{
Brian Eriksson, Gautam Dasarathy, Paul Barford, and Robert Nowak
}

\begin{abstract}
Accurate and timely identification of the router-level topology of the Internet is one of the major unresolved problems in Internet research. Topology recovery via tomographic inference is potentially an attractive complement to standard methods that use TTL-limited probes. Unfortunately, limitations of prior tomographic techniques make timely resolution of large-scale topologies impossible due to the requirement of an infeasible number of measurements. In this paper, we describe new techniques that aim toward efficient tomographic inference for accurate router-level topology measurement. We introduce methodologies based on Depth-First Search (DFS) ordering that clusters end hosts based on shared infrastructure, and enables the logical tree topology of a network to be recovered accurately and efficiently. We evaluate the capabilities of our algorithms in large-scale simulation and find that our methods will reconstruct topologies using less than $2 \%$ of the measurements required by exhaustive methods and less than $15 \%$ of the measurements needed by the current state-of-the-art tomographic approach. We also present results from a study of the live Internet where we show our DFS-based methodologies can recover the logical router-level topology more accurately and with fewer probes than prior techniques.
\end{abstract}

\section{INTRODUCTION}

Mapping the Internet's router-level topology is a compelling objective for network measurement. In addition to their appeal to network researchers, accurate and timely maps of the Internet have a wide range of applications and are of particular importance in network management, operations and security. A large number of prior studies have focused on efficient Internet router-level topology discovery using active probe-based, traceroute-like measurements e.g., [1], [2]. However, when using TTL-limited, traceroutelike measurements for reconstructing topologies, one is faced with the serious challenges of resolving anonymous routers [3] and router aliases [4]. More recent research in [5], [6] has shown how a combination of traceroute and Record Route probes can improve the accuracy of topology estimation. However, Record Route probes are also limited in that only a small percentage of Internet routers respond to the Record Route option. Finally, TTL-limited measurements are unable

B. Eriksson is with the Department of Computer Science, Boston University, Boston, MA, 02215 USA e-mail: eriksson@cs.bu.edu.

G. Dasarathy, and R. Nowak are with the Department of Electrical and Computer Engineering, University of Wisconsin - Madison.

P. Barford is with the Department of Computer Science, University of Wisconsin - Madison and Qualys.

This work was supported in part by the National Science Foundation (NSF) grants CCR-0325653, CCF-0353079, CNS-0716460 and CNS-0905186, and AFOSR grant FA9550-09-1-0140. Any opinions, findings, conclusions or other recommendations expressed in this material are those of the authors and do not necessarily reflect the view of the NSF or the AFOSR. to reveal Layer-2 hops or hops through MPLS clouds, which further reduces the accuracy of reconstructed topologies.

There are alternatives to TTL-limited measurements for Internet topology recovery. One technique that has shown promise is tomographic inference of router-level topology using end-to-end measurements of packet delay or loss. Initial work on network tomography methodologies focused on the use of multicast measurements [7], [8]. Multicast inference is attractive due to the total number of measurements necessary (i.e., probing complexity) is $O(N)$, where $N$ is the number of end hosts in the topology. However, the extremely limited deployment of open, multicast-enabled nodes renders these techniques impractical for a wide-scale topology study of the Internet. More recent work has focused on network tomography using unicast probes to obtain a measure of similarity between pairs of end hosts with respect to shared queueing delay measurements [9], [10] or shared packet loss [11]. Unfortunately, these techniques are also impractical due to the quadratic number of probes (i.e., $O\left(N^{2}\right)$ ) needed to resolve the topology.

The goal of our work is to advance the capabilities of unicast tomography such that it can be used effectively and efficiently for router-level topology discovery in the Internet. While there are a number of open challenges in the area of network tomography, the specific focus of this paper is on reducing the total number of pairwise probes that are required in order to efficiently resolve the network topology. The efficient topology reconstruction techniques presented in this paper are agnostic to the choice of unicast tomography probing methodology and depend only on how the observed pairwise characteristics conform to the underlying network topology. This approach allows for the efficient techniques described in this paper to be applied to a variety of tomographic probing approaches.

We exploit the idea of arranging end host targets in a DepthFirst Search (DFS) Order. We show how a DFS ordering clusters target end hosts based on the amount of shared infrastructure. Given this shared infrastructure clustering, we demonstrate how the observed pairwise similarity matrix from Internet measurements will have a special structure. By exploiting this matrix structure, the number of pairwise measurements necessary to resolve the logical topology of a balanced $\ell$-ary tree can be reduced from the current state-of-the-art tomography probing methodology in [12]. We then show conditions assumed by the current state-of-the-art methodologies are too restrictive and that DFS ordering can be exploited to resolve topologies using only $O(\ell N \log (N))$ pairwise measurements under a significantly less restrictive pairwise similarity conditions for a balanced $\ell$-ary tree. To the 
best of our knowledge, the resulting probing complexity of the developed DFS-based algorithms are the lowest for any unicast tomography algorithm. We believe this reduction in the number of probes is an important step toward unicast tomography being considered a feasible and practical topology discovery mechanism.

We show the performance of our DFS Ordering-based methodologies on both synthetic and real-world networks. Using simulated measurements on large-scale synthetic topologies and real-world networks via the Internet Topology Zoo [13] that satisfy our pairwise similarity conditions, we show our methodologies require fewer than $2 \%$ of the pairwise measurements required by exhaustive methods and $15 \%$ less than current state-of-the-art techniques. Finally, we present results using real-world delay-based tomographic probes which show that the techniques presented here reconstruct logical tree topologies more accurately and using fewer pairwise probes than prior developed methodologies.

The remainder of the paper is structured as follows. In Section II, we describe previous tomographic methods for Internet logical topology discovery and other related work. The topology discovery problem and the concept of DFS Ordering are introduced in Section III. In Section IV, an efficient logical topology discovery algorithm is described using a known DFS Ordering of the end hosts. Given that real world topologies will not have the DFS ordering known, in Section V we show how a DFS ordering of the end hosts can be found from relatively few topology measurements under a specified $\delta$ Margin Condition on the observed similarities between end hosts. In Section VI we show how the DFS ordering can be resolved when only a less restrictive Monotonic Condition is considered on the observed pairwise similarities. Finally, in Section VII the results of our experiments on both synthetic and real world topologies show the improvements of our procedure for estimating the logical topology of a network over previous techniques. We conclude and describe future work in Section VIII.

\section{RELATED WORK}

The initial work most directly related to the research in this paper is the application of bottom-up agglomerative clusteringbased methodologies [14] explored in [7], [8], [15] for use on Internet topology reconstruction. Bottom-up agglomerative clustering resolves the hierarchical clustering of a set of objects with pairwise similarity values by finding the maximum similarity element and merging the rows/columns of the similarity matrix corresponding to those two end hosts, then finding the next maximum element and merging those rows/columns of the similarity matrix to the new maximum element. This process is repeated until all the rows/columns are merged and the hierarchical clustering of the entire set of objects is resolved. In terms of network tomography, this method requires obtaining the entire similarity matrix (e.g., $O\left(N^{2}\right)$ pairwise probes given $N$ number of end hosts in the topology). The agglomerative clustering methodology will be considered the worst case probing bounds, as it performs an exhaustive probing of every possible pair of end hosts in the network. The large number of probes required is due to the decoupling of topology measurements and topology inference, where no information from prior measurements is used to inform new measurements, and topology inference is performed completely separate from the measurement process.

A more efficient probing methodology is the Sequential Topology Inference algorithm from [12]. This work iteratively builds the logical tree structure and leverages the current estimated logical tree structure to determine where the next probe pair measurements should be performed. This couples topology inference and measurement into one process by exploiting the tree structure of the topology. For a balanced $\ell$ ary tree (a balanced tree where each non-leaf node has exactly $\ell$ children), this reduces the number of probes needed from $O\left(N^{2}\right)$ for agglomerative clustering, to at most $\ell N \log _{\ell}(N)$. In Sections V and VI, we show how improvements to this performance can be obtained by exploiting the structure of not just the tree topology, but the structure of the topology measurements. We show how our methodologies can further reduce the number of probes compared to this current state-of-the-art. Additionally, the Sequential Topology methodology requires strict conditions on the observed pairwise similarities, where each router induces a known minimum amount of observed similarity between pairs of end hosts. Our methodology presented in Section VI will demonstrate an efficient tomography technique that requires significantly less restrictive conditions on the observed pairwise similarity values.

We introduce two targeted measurement methodologies based on a Depth-first Search (DFS) of a topology. For a collection of end hosts in a tree topology, any of the nonunique ordinal lists found from a depth-first search on the leaf nodes of a tree structure (considered here as end hosts) can be defined as a DFS ordering. This can also be considered a topological sort [16] on only the end hosts of a logical topology. The idea of topological sort has been explored previously in sensor network literature in [17], where a topological sort of the nodes in a sensor network provides efficient routes through the network with low power consumption. Due to the focus on wire-line networks in this paper, we are not able to choose the routing. Instead we will use a modified version of topological sorting to efficiently reconstruct the logical routing from observed Internet measurements.

The work presented here is an extension of the authors' work in [18]. While the prior conference paper only contained the margin-based DFS approach described in Section V, the work here expands upon that technique to develop a new tomography methodology that requires a significantly less restrictive condition on how observed pairwise similarities conform to the underlying network topology in Section VI.

\section{Depth-First SeARch (DFS) ORdering}

Consider two end hosts in the network, denoted as $x_{i}, x_{j}$. Through a probing mechanism we have the ability to observe pairwise similarity between these end hosts, denoted as $s_{i, j}$ (e.g., a measure of delay covariance or shared packet loss between two end hosts). Instead of examining the probing technique used to generate the similarities, we will focus on 
specific conditions with respect to how these pairwise similarities conform to the underlying network tree topology structure. Specifically, we examine how the similarities conform to the logical topology shared path length, $p_{i, j}$, the number of logical routers shared between end hosts $x_{i}$ and $x_{j}$ in the paths from the root node to the two end hosts.

The foundation for this work is the idea of a Depth-First Search (DFS) Ordering of the end hosts. A depth-first search (DFS) is a tree search that starts at the tree root and progresses down the tree labeling each node and backtracking only when a node has been explored fully (e.g., every child of that node has been labeled). We formally define a DFS Ordering as any ordinal list of the end hosts (which will be considered the leaf nodes of the logical routing tree) that would satisfy the ordering found by a depth-first search of that logical tree structure ignoring the labeling of the internal nodes of the tree.

Definition 1: A DFS Ordering, $\pi_{D F S}:\{1,2, \ldots, N\} \rightarrow$ $\{1,2, \ldots, N\}$, enumerates the end hosts such that there exists a depth-first search on the tree structure $\mathcal{T}$ that would reveal the items in the order of $\pi_{D F S}$.

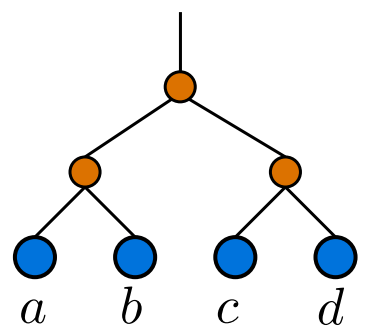

Fig. 1. Example simple logical topology in a DFS Ordering.

For the tree structure in Figure 2, we can find the following DFS orderings all of which would satisfy some depth-first search on the tree topology:

$$
\begin{array}{llll}
\{a, b, c, d\} & \{a, b, d, c\} & \{b, a, c, d\} & \{b, a, d, c\} \\
\{c, d, a, b\} & \{d, c, a, b\} & \{c, d, b, a\} & \{d, c, b, a\}
\end{array}
$$

There are also many possible end host enumerations that would violate a DFS ordering of the tree. For example, the ordering $\{a, c, d, b\}$ does not satisfy a depth-first search of the end hosts for the tree structure in Figure 1.

The power of a depth-first search can be seen when examining the logical topology shared path matrix P. For a DFS ordering of the topology in Figure 2 (e.g., $\{a, b, c, d\})$, the ordered shared path matrix $\mathbf{P}^{D F S}$ will be found as:

$$
\mathbf{P}^{D F S}=\left[\begin{array}{c|cccc} 
& a & b & c & d \\
\hline a & 2 & 2 & 1 & 1 \\
b & 2 & 2 & 1 & 1 \\
c & 1 & 1 & 2 & 2 \\
d & 1 & 1 & 2 & 2
\end{array}\right]
$$

Where $p_{a, b}^{D F S}=2$, indicates the two paths from the root to end hosts $a, b$ (in Figure 1) share two common logical routers.

For an enumeration that does not satisfy a DFS ordering (e.g., $\{a, c, d, b\}$ ), the out-of-order shared path matrix $\left(\mathbf{P}^{\text {NotDFS }}\right)$ will be found as:

$$
\mathbf{P}^{\text {NotDFS }}=\left[\begin{array}{c|cccc} 
& a & c & d & b \\
\hline a & 2 & 1 & 1 & 2 \\
c & 1 & 2 & 2 & 1 \\
d & 1 & 2 & 2 & 1 \\
b & 2 & 1 & 1 & 2
\end{array}\right]
$$

Using a set of end hosts in a DFS ordering, we can state the following proposition,

Proposition 1: Given a set of end hosts $\left\{x_{1}, x_{2}, \ldots, x_{N}\right\}$ in a DFS Ordering, the resulting shared path matrix $\mathbf{P}$ has the following structure:

$$
p_{i, i+1} \geq p_{i, i+k} \quad: \text { for all } k=\{1,2, \ldots, N-i\}
$$

For all $i=\{1,2, \ldots, N-1\}$.

Proof: Consider the case where the end hosts are in a DFS ordering, but $p_{i, i+j}<p_{i, i+k}$ (for some $0 \leq j<k$ ). This states that end hosts $x_{i}, x_{i+k}$ have more shared infrastructure than $x_{i}, x_{i+j}$ (i.e., a longer shared path length from the root). This implies the tree structure has $x_{i}$ and $x_{i+k}$ connected to an ancestor router at some point of depth (i.e., level of shared infrastructure), while $x_{i+j}$ is connected at some point in the tree structure shallower in comparison to $x_{i}$ (i.e., at some level with less shared infrastructure than $x_{i}$ and $\left.x_{i+k}\right)$. But by the depth-first search ordering, this requires $j>k$ as a depth-first search would encounter $x_{i+k}$ before $x_{i+j}$, thus violating the setup of the problem. Therefore, if the end hosts are in a DFS ordering, Proposition 1 must hold.

\section{LOGICAl TOPOlOgy Discovery USING DFS ORDERING}

Assume that all the end hosts in an unknown topology are already in a DFS ordering. Given this ordering, we look to reconstruct the logical topology using fewer than all possible pairwise similarities. To begin, we assume that the observed similarity measurements satisfy the Monotonic Condition with respect to the underlying network tree structure.

Condition 1: The observed pairwise similarity matrix $\mathbf{S}$ and shared path matrix $\mathbf{P}$ satisfy the Monotonic Condition if for all end hosts $i, j, k$ where the tree topology shared path satisfies $p_{i, j}>p_{j, k}$, then the observed pairwise similarity satisfies $s_{i, j}>s_{j, k}$.

Using the results from Proposition 1 and the Monotonic Condition, we can state that the similarity matrix $\mathbf{S}$ has structure directly relating to the structure of the shared path matrix $\mathbf{P}$.

Proposition 2: Given a set of end hosts $\left\{x_{1}, x_{2}, \ldots, x_{N}\right\}$ in a DFS Ordering, with the similarity matrix $\mathbf{S}$ satisfying the Monotonic Condition, then the similarity matrix $\mathbf{S}$ will have the following property:

$$
s_{i, i+1} \geq s_{i, i+k} \quad: \text { for all } k=\{1,2, \ldots, N-i\}
$$

For all $i=\{1,2, \ldots, N-1\}$.

Proof: Given Proposition 1 and the Monotonic Condition, this property of the similarity matrix $\mathbf{S}$ follows.

Using the results of this proposition, we can now devise an efficient logical tree reconstruction procedure, the ORDEREDTOPO method in Algorithm 1 for a set of end hosts in a DFS 
ordering with pairwise similarities satisfying the Monotonic Condition. An example implementation of this procedure can be seen in Figure 2 and bounds on the number of pairwise similarities required are declared in Theorem 1.

Algorithm 1 - DFS Ordered Logical Topology Discovery Algorithm - OrderedTopo $(\mathbf{X}, \mathbf{S})$

\section{Given:}

- Set of $N$ end hosts in a DFS ordering, $\mathbf{X}=$ $\left\{x_{1}, x_{2}, \ldots, x_{N}\right\}$

- Set of observed pairwise similarities for end hosts in a DFS ordering, $\left\{s_{1,2}, s_{2,3}, \ldots, s_{N-1, N}\right\}$

Main Body:

- Set of tree nodes, $\widehat{V}=\left\{x_{1}, x_{2}, \ldots, x_{N}\right\}$.

- Set of tree edges, $\widehat{E}=\emptyset$.

- Reconstructed tree topology, $\widehat{\mathcal{T}}=(\widehat{V}, \widehat{E})$.

- Set of merge nodes $Y=\left\{x_{1}, x_{2}, \ldots, x_{N}\right\}$.

- For $k=\{1,2, \ldots, N-1\}$

1) Find $\widehat{j}=\operatorname{argmax}_{j=\left\{1,2, \ldots,\left|V^{\prime}\right|-1\right\}} s_{j, j+1}$.

2) Create new interior node, $x^{*}$.

3) Add new interior node to the set of vertices, $\widehat{V}=$ $\widehat{V} \cup x^{*}$.

4) Add new edges to the new interior node, $\widehat{E}=$ $\widehat{E} \bigcup\left\{Y_{\widehat{j}}, x^{*}\right\} \bigcup\left\{Y_{\widehat{j}+1}, x^{*}\right\}$.

5) Find the set of merge nodes that reference the old interior nodes,

$$
\mathcal{I}=\left\{i: Y_{i}=Y_{\widehat{j}} \text { or } Y_{i}=Y_{\widehat{j}+1}\right\}
$$

6) Update the merge nodes, $Y_{i}=x^{*}$ for all $i \in \mathcal{I}$.

7) Update the similarity values to avoid choosing this similarity value again, set $s_{\widehat{j}, \widehat{j}+1}=0$.

\section{Output:}

1) Return estimated tree topology, $\widehat{\mathcal{T}}$

Theorem 1: Using the set of end hosts in a DFS Ordering and pairwise similarities satisfying the Monotonic Condition, only $N-1$ pairwise probes (the similarity values $s_{i, i+1}$ for $i=\{1,2, \ldots, N-1\})$ are needed to reconstruct the unknown logical tree topology using the ORDEREDTOPO Algorithm.

Proof: For each end host, bottom-up agglomerative clustering requires only knowledge of which other end host has the most shared topology. Given the Monotonic Condition, this is equivalent to finding the pair of end hosts with the largest pairwise similarity magnitude. Unfortunately, to acquire this knowledge, it was previously necessary to obtain all possible similarity values (on the order of $O\left(N^{2}\right)$ pairs for $N$ end hosts). Given both a DFS ordering of the end hosts and the Monotonic Condition, the only similarity values necessary to infer the logical topology will be the value of the immediately preceding end host similarity $\left(s_{i-1, i}\right)$ and the immediately successive end host similarity $\left(s_{i, i+1}\right)$ for each end host $x_{i}$, with $i=\{1,2, \ldots, N\}$. This is due to the Proposition 2 stating that the similarity $s_{i, i+1} \geq s_{i, i+j}$ for any $j>1$. Therefore, end host $x_{i}$ will share the most infrastructure in the topology with either $x_{i+1}$ or $x_{i-1}$. In order to reconstruct the tree topology, only the similarity values associated with these consecutive pairs of end hosts are needed. The magnitude of these two similarity values $\left(s_{i-1, i}, s_{i, i+1}\right)$ will directly inform us as to the structure of the logical topology using a modified bottomup agglomerative clustering procedure that only considers this subset of pairwise similarities, which is the ORDEREDTOPO Algorithm.

\section{MARGIN-BASED DFS ORDERING Estimation}

A limitation of the methodology in the ORDEREDTOPO Algorithm is the assumption that the end hosts are already correctly arranged in a depth-first search (DFS) ordering. In any non-trivial problem, this ordering will not be known. Instead, given no prior knowledge of the topology, we must estimate a DFS Ordering from targeted pairwise similarity measurements. Influenced by the work in [12], in this section we require a more restrictive assumption on the similarities than the Monotonic Condition. We assume the observed tomographic measurements conform to the $\delta$-Margin Condition.

Condition 2: The observed similarity matrix $\mathbf{S}$ and shared path matrix $\mathbf{P}$ satisfy the $\delta$-Margin Condition if for all end hosts $i, j, k$, the observed similarities satisfy $s_{i, j}>s_{j, k}+\delta$ (for some specified $\delta>0$ ) if and only if the tree topology shared path satisfies $p_{i, j}>p_{j, k}$.

Remark: Delay-based unicast methods exploit the observation of shared queuing delay between pairs of end hosts. Consider the value $\delta$ to be the minimum queueing delay induced by a router in the network topology. Note that the Monotonic Condition is a boundary condition where $\delta=0$ and the reverse implication does not need to be satisfied $\left(s_{i, j}>s_{j, k}\right.$ implies $\left.p_{i, j}>p_{j, k}\right)$. Therefore, due to the significantly weaker assumptions, any tree reconstruction methodology that holds under the Monotonic Condition will also hold under the $\delta$ Margin Condition for any $\delta$.

The intuition behind our margin-based DFS ordering methodology is as follows. Given a random ordering of the set of end hosts, consider choosing a single end host $\left(x_{1}\right)$ and obtaining the pairwise similarities between this end host and all other end hosts in the set $\left(=\left\{s_{1,2}, s_{1,3}, s_{1,4}, \ldots, s_{1, N}\right\}\right)$. Some end hosts will have very high pairwise similarity, while others will have significantly less shared infrastructure with the chosen end host and therefore have low pairwise similarity. By sorting these obtained similarity values, this would place the end hosts that have more shared infrastructure at one end of the list, and the end hosts with little shared infrastructure at the other end of the list. We define ordering with respect to a single end host as the partial ordering, $\pi$, of the set of end hosts, such that $\pi:\{2,3, \ldots, N\} \rightarrow\{2,3, \ldots, N\}$ (with $s_{1, \pi_{i}} \geq s_{1, \pi_{i+j}}$ : for all $j \geq 1$ ). This partial ordering cannot be considered a DFS ordering for the reasons shown in Figure 3-(Left). While a significant fraction of the end hosts will have observed pairwise similarity within some margin $\delta$ when compared against the chosen end host, as seen in Figure 3-(Center), a DFS ordering inside this cluster is unknown using only this single end host vantage point.

This implies that pairwise similarities from more than a single end host vantage point will be required to correctly order the entire set of end hosts. From the example Figure 3- 

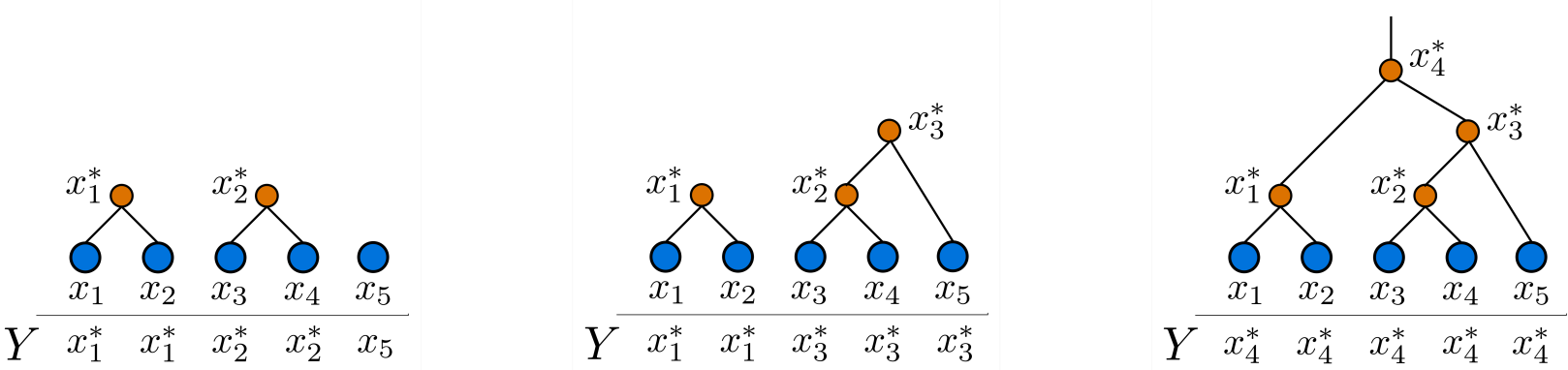

Fig. 2. Example implementation of the ORDEREDTOPO Algorithm annotated with merge node values for each leaf node, $Y$. (Left) - Intermediate reconstructed tree with two interior nodes, $x_{1}^{*}, x_{2}^{*}$; (Center) - Addition of another interior node $x_{3}^{*}$ given maximum similarity value of $s_{4,5}$, (Right) - Final interior node $x_{4}^{*}$ added to complete the tree structure.
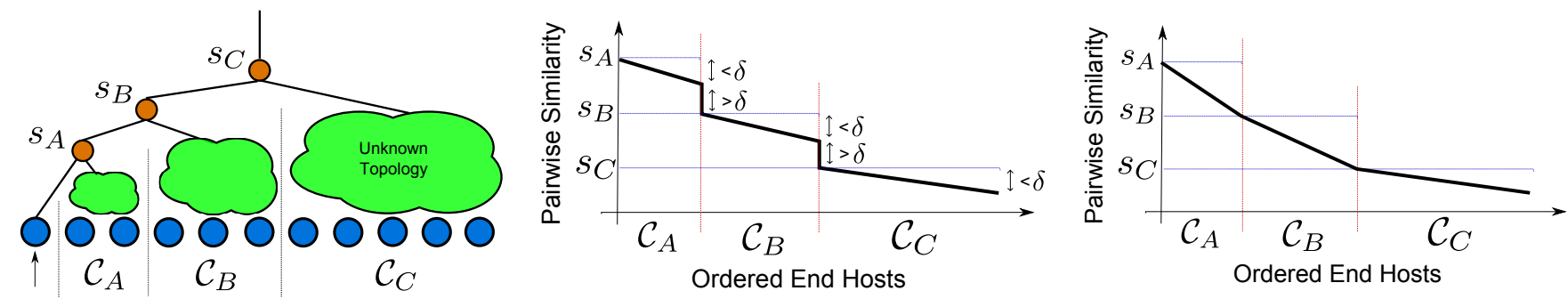

Fig. 3. End Host Ordering based on condition - (Left) Example of similarity values from a single end host revealing partial ordering, (Center) - Resulting ordered pairwise similarity values given the $\delta$-Margin Condition, (Right) - Resulting ordered pairwise similarity values given the Monotonic Condition.

(Center), consider that any similarity will be within a $\delta$ deviation of one of three values $\left\{s_{A}, s_{B}, s_{C}\right\}$. Having correctly ordered the end hosts based on these three clusters, we now look to order the subclusters (e.g., what should the ordering be of all the end hosts with similarity within a $\delta$ deviation of $s_{C}$ for the topology?). One could consider dividing the set of end hosts into similarity clusters and for each cluster repeating this probing process. This would be performed by taking a new intracluster vantage point (i.e., randomly choosing a new end host to compare against), and then reordering the subset of intracluster end hosts based on the pairwise similarity values with this new vantage point. Therefore, we look to a recursive methodology that at each iteration bisects the ordered set of end hosts into two topologically significant clusters. This reduces our objective to the problem of finding the correct end host to bisect the set of end hosts at each iteration of the algorithm.

The simplest approach to this bisection problem is, for a given margin value $\delta$, sorting the similarity values and finding all the possible bisection candidate end hosts. We denote the set $\mathcal{I}$, where $i \in \mathcal{I}$ if the similarity difference between the partial ordered $i$-th (denoted as $\pi_{i}$ ) and the partial ordered $(i+1)$-th (denoted as $\left.\pi_{i+1}\right)$ similarity value is more than $\delta$, thereby indicating a topology difference between the two end hosts with respect to end host $x_{1}$,

$$
\mathcal{I}=\left\{i: s_{1, \pi_{i}}-s_{1, \pi_{i+1}}>\delta\right\}
$$

The bisection point will be the end host $i^{*} \in \mathcal{I}$ that results in the two bisected end host sets $\mathbf{X}_{A}=\left[x_{1}, x_{\pi_{1}}, x_{\pi_{2}}, \ldots, x_{\pi_{i^{*}}}\right]$ and $\mathbf{X}_{B}=\left[x_{\pi_{i^{*}+1}}, \ldots, x_{\pi_{N}}\right]$ to be closest in size to each other among all choices of $i^{*} \in \mathcal{I}$.

$$
i^{*}=\underset{i \in \mathcal{I}}{\arg \min }\left|\pi_{i}-\frac{N}{2}\right|
$$

Using this intuition, we present the MARGINORDER method in Algorithm 2 to find a DFS Ordering for a set of end hosts using this recursive bisection methodology.

Proposition 3: Using the MARGINORDER Method, the number of pairwise similarities measurements needed to correctly obtain a DFS Ordering for a balanced $\ell$-ary tree (where each non-leaf node has $\ell$ children) satisfying the $\delta$-margin condition with $N$ end hosts is upper bounded by $p(\ell) N \log _{\ell} N$ probe pairs (where $p(\ell)=\left(\frac{\ell+1}{2}-\frac{1}{\ell}\right)$ ).

Proof: Proof of this proposition is found in the appendix.

The MARGINTOPOLOGY topology reconstruction methodology consists of finding a DFS ordering of the end hosts using the MARginOrder Algorithm, and then resolving the logical topology using the ORDEREDTOPOLOGY Algorithm. Using Proposition 3 and Theorem 1, we bound the total number of pairwise probes required by the margin-based topology reconstruction methodology. Note that the MARGINTOPOLOGY topology reconstruction methodology has a pairwise probing upper bound that requires fewer pairwise similarities than the current state-of-the-art efficient tomography approach in [12], which also requires the $\delta$-Margin Condition on the pairwise similarities.

Theorem 2: Using the MARGINTOPOLOGY algorithm, the logical topology for a balanced $\ell$-ary tree with $N$ end hosts can be reconstructed requiring at most $N\left(p(\ell) \log _{\ell} N+1\right)$ pairwise similarities that satisfy the $\delta$-Margin Condition.

Proof: Proof of this theorem follows from Proposition 3 and Theorem 1. 
Algorithm 2 - Margin-Based DFS Ordering Algorithm MarginOrder $(\mathbf{X}, \mathbf{S}, \delta)$

\section{Given:}

- Unordered set of $N$ end hosts with unknown logical topology $\mathbf{X}=\left\{x_{1}, x_{2}, \ldots, x_{N}\right\}$

- Pairwise similarity matrix, $\mathbf{S}$

- Margin condition, $\delta>0$

\section{Main Body:}

1) Find the pairwise similarity values with respect to end host $x_{1},\left\{s_{1,2}, s_{1,3}, \ldots, s_{1, N}\right\}$.

2) Sort the set of pairwise similarities, obtaining the partial ordering with respect to end host $x_{1}, \pi:\{2,3, \ldots, N\} \rightarrow$ $\{2,3, \ldots, N\}$.

3) Find $\mathcal{I}$, the set of indices where the difference between consecutive sorted similarity values is greater than $\delta$.

$$
\mathcal{I}=\left\{i: s_{1, \pi_{i}}-s_{1, \pi_{i+1}} \geq \delta\right\}
$$

4) Bisect the set of sorted end hosts $\mathbf{X}$ at the index of $i^{*}$ that creates two sets most equal in size using Equation 1, creating sorted end host subsets $\mathbf{X}_{A}=\left\{x_{1}, x_{\pi_{1}}, \ldots, x_{\pi_{i^{*}}}\right\}$, $\mathbf{X}_{B}=\left\{x_{\pi_{i^{*}+1}}, \ldots, x_{\pi_{N-1}}\right\}$.

5) If $\left|\mathbf{X}_{A}\right|>2$, then find $\mathbf{X}_{A}=\operatorname{MarginOrder}\left(\mathbf{X}_{A}, \mathbf{S}\right.$, $\delta)$

6) If $\left|\mathbf{X}_{B}\right|>2$, then find $\mathbf{X}_{B}=\operatorname{MarginOrder}\left(\mathbf{X}_{B}, \mathbf{S}\right.$, $\delta)$

\section{Output:}

1) Return the reordered indices of the end hosts, $\mathbf{X}=$ $\left[\mathbf{X}_{A} \mathbf{X}_{B}\right]$

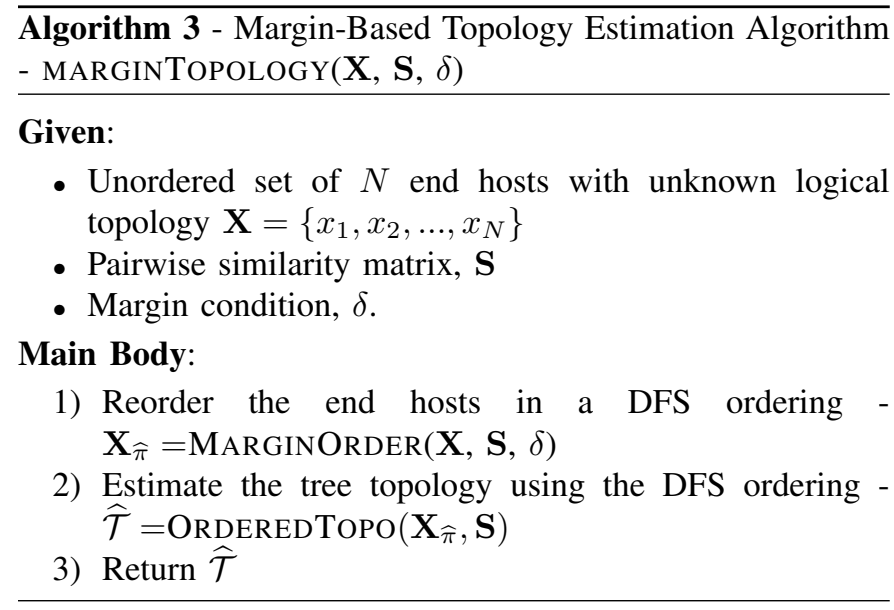

\section{Monotonic-Based DFS Ordering Estimation}

In many real world tomography problems, the $\delta$-Margin Condition will be too restrictive. Instead, we look to efficiently reconstruct the tree topology when only the Monotonic Condition holds, where for any triple of end hosts $x_{i}, x_{j}, x_{k}$, if the shared path values satisfy $p_{i, j}>p_{j, k}$, then the pairwise similarities satisfy $s_{i, j}>s_{j, k}$. Our ordering estimation methodology begins similar to the margin-based approach where the ordering is found by a recursive bisection of the set of end hosts, but we also require a small number of additional pairwise similarities to reinforce each bisection choice due to uncertainties introduced by the Monotonic Condition.

Given a random ordering of the set of end hosts $\left(\left\{x_{1}, x_{2}, \ldots, x_{N}\right\}\right)$, consider choosing a single end host $\left(x_{1}\right)$ and obtaining the similarity measurements between this and all other hosts in the set $\left(=\left\{s_{1,2}, s_{1,3}, \ldots, s_{1, N}\right\}\right)$ to find the partial ordering, $\pi$. Similar to the methodology in Section V, we look to reduce the total number of pairwise measurements needed by bisecting the partial order $\pi$ and repeatedly taking pairwise measurements only with respect to each bisected subset. While the $\delta$-Margin Condition allows us to easily find the bisection point $x_{i}^{*}$ through the use of the similarity difference (where, $s_{1, \pi_{i}}-s_{1, \pi_{i+1}}>\delta$ ), under the Monotonic Condition this deviation will not indicate changes in the tree topology, this can be seen in Figure 3-(Right). Therefore, we must devise a different methodology for finding the bisection point that represents a split in the tree, as a large similarity difference will not necessarily imply a topology change assuming only the Monotonic Condition holds on the pairwise similarity values.

Imagine performing bottom-up agglomerative clustering on the set of partial ordered end hosts. Pairs of end hosts are repeatedly merged together until all are combined into a single cluster by the final merge operation. Prior to the final merge operation, the set of ordered end hosts are bisected into two sets separated by a split in the tree topology. When all the end hosts are in a DFS ordering, this split can be defined by a single point that bisects these two sets in the ordering, $x^{*}$. Using standard bottom-up agglomerative clustering, this would require examination of all possible pairwise similarity values (on the order of $N^{2}$ for $N$ end hosts). By exploiting the partial ordering of the end hosts, $\pi$, this split can be found using a modified agglomerative clustering procedure which requires significantly fewer than all the possible pairwise similarities.

Consider performing agglomerative clustering on only a subset of $m$ end hosts evenly spaced in the partial ordering (e.g., such that there are $\frac{N}{m}$ between each agglomerative clustering end host) in Figure 4-(Left). The final merge of the agglomerative clustering on these $m$ end hosts (i.e., Figure 4(Center)) will reveal which subset of $\frac{N}{m}$ hosts that contain the bisection point $x^{*}$. Once this subset is revealed, we choose $m$ new end hosts inside this subset of $\frac{N}{m}$ (i.e., the set $\mathbf{X}_{R}$ in Figure 4-(Right)) and again perform agglomerative clustering to find a subset of interest, this time of size $\frac{N}{m^{2}}$ (again, containing the bisection point $x^{*}$ ). This process is repeated until the subset of interest is of size less than or equal to $m$, where our modified agglomerative clustering will resolve the top most split in the tree and bisection point $x^{*}$.

This recursive bisection algorithm, MONOTONICBISECT, is stated in Algorithm 4. The power of this methodology is found by the distillation of agglomerative clustering measurements, requiring at most $m^{2} \log _{m} N$ pairwise measurements, while standard agglomerative clustering would require $O\left(N^{2}\right)$ pairwise measurements.

This recursive methodology is only to find the bisection object at a single iteration of the ordering algorithm. It must be repeated multiple times to find a true DFS ordering on the end hosts. The complete methodology for finding the DFS ordering under the monotonic condition is described in the 

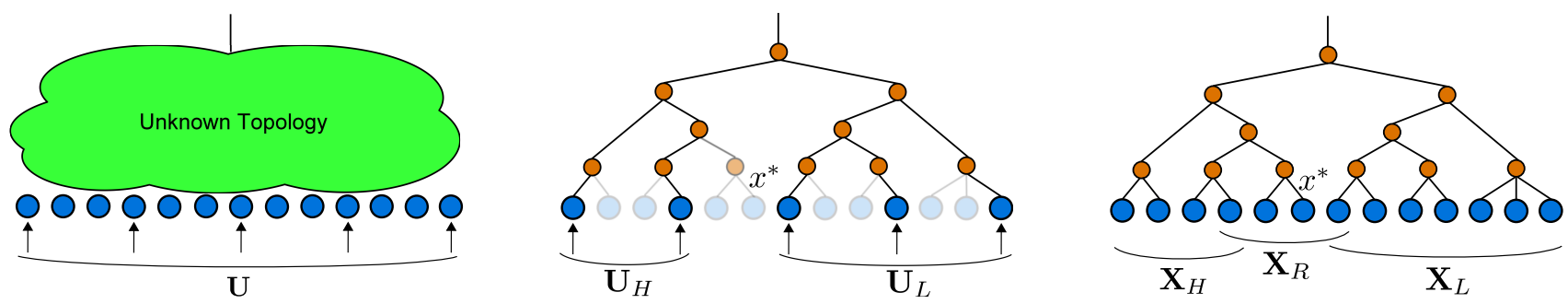

Fig. 4. (Left) Set of end hosts in partial order, with subset of end hosts $U$ given $m=5$. (Center) Tree structure found through agglomerative clustering on the set $U$, with top-level split partitioning into $U_{H}, U_{L}$. (Right) Resulting end host sets $X_{R}, X_{L}, X_{H}$.

\begin{tabular}{l}
\hline Algorithm 4 - Monotonic-Based Bisection Algorithm - \\
MOnOTOnicBisect $(\mathbf{X}, \mathbf{S}, m)$
\end{tabular}

\section{Given:}

- Set of $\mathbf{N}$ end hosts in partial order $\mathbf{X}=\left\{x_{1}, x_{2}, \ldots, x_{N}\right\}$

- Pairwise similarity matrix, $\mathbf{S}$

- Number of clustering hosts, $m$

\section{Main Body:}

1) Pick a subset of end host indices $\mathbf{U} \subset\{1,2, \ldots, N\}$ such that $|\mathbf{U}|=m$ and these indices are uniformly spaced in $\{1,2, \ldots, N\}$.

2) Using bottom-up agglomerative clustering, reconstruct the tree structure of the subset of end hosts indexed by U.

3) Find the last merge of the agglomerative clustering algorithm, between end host subsets $\mathbf{U}_{L}, \mathbf{U}_{H}$ (such that $\mathbf{U}_{L} \cup \mathbf{U}_{H}=\mathbf{U}$ and $\mathbf{U}_{L} \bigcap \mathbf{U}_{H}=\emptyset$ ).

4) Define the largest similarity in the 'lower' set $s_{L}^{\max }=$ $\max _{i \in \mathbf{U}_{L}} s_{1, i}$, related to end host $x_{L}^{\max }$. And the smallest similarity in the 'higher' set, $s_{H}^{\text {min }}=\min _{i \in \mathbf{U}_{H}} s_{1, i}$, related to end host $x_{H}^{\min }$.

5) Divide the set $X$ into three subsets: the 'higher' set $\mathbf{X}_{H}=\left\{x_{i}: s_{1, i} \geq s_{H}^{m i n}\right\}$, the 'lower' set $\mathbf{X}_{L}=$ $\left\{x_{i}: s_{1, i} \leq s_{L}^{\max }\right\}$ and the 'residual' set $\mathbf{X}_{R}=$ $\left(\mathbf{X} \backslash\left(\mathbf{X}_{L} \cup \mathbf{X}_{H}\right)\right) \bigcup\left\{x_{L}^{\max }, x_{H}^{\min }\right\}$.

6) If $|\mathbf{X}| \leq m$, set $x^{*}=x_{H}^{\text {min }}$

7) Else, set $x^{*}=$ MOnotonicBisect $\left(\mathbf{X}_{R}, \mathbf{S}, m\right)$

\section{Output:}

1) Return $x^{*}$.

\section{MONOTONICORDER methodology in Algorithm 5.}

Finally, we combine both the MONOTONICORDER and the ORDEREDTOPOLOGY algorithms in order to resolve the logical topology from pairwise similarities in the MONOTONICTOPOLOGY algorithm. Bounds on the number of pairwise similarities required to resolve the topology are derived in Theorem 3.

Theorem 3: Using the MONOTONICTOPOLOGY algorithm, the logical topology for a balanced $\ell$-ary tree with $N$ end hosts can be reconstructed requiring at most $N\left((\ell+9) \log _{2} N+1\right)$ pairwise similarities that satisfy the monotonic condition.

Proof: Proof of this proposition is found in the appendix.
Algorithm 5 - Monotonic-Based DFS Ordering Algorithm MONOTONiCORder $(\mathbf{X}, \mathbf{S}, \mathrm{m})$

\section{Given:}

- Unordered set of $N$ end hosts with unknown logical topology $\mathbf{X}=\left\{x_{1}, x_{2}, \ldots, x_{N}\right\}$

- Pairwise similarity matrix, $\mathbf{S}$

- Number of clustering nodes, $m$.

\section{Main Body:}

1) Find the pairwise similarity values with respect to end host $x_{1},\left\{s_{1,2}, s_{1,3}, \ldots, s_{1, N}\right\}$.

2) Sort the set of pairwise similarities, obtaining the partial ordering with respect to end host $x_{1}, \pi:\{2,3, \ldots, N\} \rightarrow$ $\{2,3, \ldots, N\}$.

3) Using the Monotonic Condition Bisection algorithm, MONOTONICBISECT (Algorithm 4), find the bisection index, $i^{*}$.

4) Create sorted end host subsets $\mathbf{X}_{A}=$ $\left\{x_{1}, x_{\pi_{1}}, \ldots, x_{\pi_{i^{*}}}\right\}, \mathbf{X}_{B}=\left\{x_{\pi_{i^{*}+1}}, \ldots, x_{\pi_{N-1}}\right\}$.

5) If $\left|\mathbf{X}_{A}\right|>2$, then find $\mathbf{X}_{A}=\operatorname{MonotonicORder}\left(\mathbf{X}_{A}\right.$, $\mathrm{S}, \mathrm{m})$

6) If $\left|\mathbf{X}_{B}\right|>2$, then find $\mathbf{X}_{B}=\operatorname{MonotonicORdeR}\left(\mathbf{X}_{B}\right.$, $\mathrm{S}, \mathrm{m})$

\section{Output:}

1) Return the reordered indices of the end hosts $\mathbf{X}=$ $\left[\mathbf{X}_{A} \mathbf{X}_{B}\right]$.

Algorithm 6 - Monotonic-Based Topology Estimation Algorithm - MONOTONicTopology $(\mathbf{X}, \mathbf{S}, m)$

\section{Given:}

- Unordered set of $N$ end hosts with unknown logical topology $\mathbf{X}=\left\{x_{1}, x_{2}, \ldots, x_{N}\right\}$

- Pairwise similarity matrix, $\mathbf{S}$

- Number of clustering nodes, $m$.

Main Body:

1) Reorder the end hosts in a DFS ordering $\mathbf{X}_{\widehat{\pi}}=$ MonotonicOrder $(\mathbf{X}, \mathbf{S}, m)$

2) Estimate the tree topology using the DFS ordering $\widehat{\mathcal{T}}=\operatorname{OrDEREDTOPO}\left(\mathbf{X}_{\widehat{\pi}}, \mathbf{S}\right)$

3) Return $\widehat{\mathcal{T}}$

\section{EXPERIMENTS}

To assess performance of our efficient network tomography methodologies, we perform experiments on both synthetic and 
real-world Internet topologies.

\section{A. Prior Methods}

1) Agglomerative Clustering: Consider having knowledge of every pairwise similarity value for all $N$ end hosts in the topology. Given this similarity matrix we would know which set of end hosts have the largest similarity in the entire topology, and hence, know which set of end hosts have the most shared infrastructure from the root node. For the bottom-up Agglomerative Clustering algorithm (applied in a networking context in [7], [8], [15]), at each step of the algorithm the current set of end hosts with the largest similarity are found, and a logical router is inserted connecting this set of end hosts together. The corresponding rows/columns in the similarity matrix for these two end hosts are then merged together. This process is repeated until there are no more rows/columns in the matrix left to merge. The main disadvantage of this methodology is that it requires knowledge of all $\frac{N(N-1)}{2}$ similarity values, which is effectively exhaustive probing of all the end hosts in the network.

2) Sequential Logical Topology Reconstruction: Informed by the generic tree structure of the topology, the work in [12] shows that the number of probes needed to reconstruct the topology can be considerably reduced when the observed similarities satisfy the $\delta$-Margin Condition. This methodology depends upon sequentially building the tree topology for each end host. For a given end host, $x_{i}$, the pairwise similarity for this end host and representative end hosts for the children of the root node are found. Given the child of the root node with the largest similarity (and thus the most shared topology), $c_{i}^{*}$, the pairwise similarity is found between the end host and the children of the specified child $\left(s_{i, c_{i}^{*}}\right)$. This similarity value and margin value, $\delta$, determines whether the end host is a sibling, child, or descendant of $c_{i}^{*}$. This process is repeated until the leaf node with the largest pairwise similarity is found. On a balanced $\ell$-ary tree (a balance tree where each non-leaf node has $\ell$ children), each end host requires at most $\ell \log _{\ell} N$ pair probes, thus for the entire topology the number of pairwise probes needed is upper bounded by $\ell N \log _{\ell} N$.

Table I compares the probing upper bounds for all four topology reconstruction methodologies (agglomerative clustering, sequential, MARGINTOPOLOGY, and MONOTONICTOPOLOGY). The MARGINTOPOLOGY algorithm has the smallest probing complexity upper bound of all algorithms, but requires the restrictive $\delta$-Margin Condition on the observed similarity values. In comparison with the Sequential Topology algorithm, which also requires the $\delta$-Margin Condition, from Equation 4 we can see that $\frac{p(\ell)}{\ell} \leq 0.5625$ for all choices of $\ell$, therefore the MARGINTOPOLOGY will require fewer pairwise probes than the Sequential Topology algorithm for any feasible $\ell, N$ topologies. The MONOTONICTOPOLOGY algorithm has upper bounds requiring more probes than the two margin-based methodologies, but will be guaranteed to succeed when the similarity margin $\delta$ is not required on the observed pairwise similarities. The MONOTONICTOPOLOGY algorithm also requires significantly fewer pairwise probes than the agglomerative clustering methodology, which is the only other methodology that is also guaranteed to reconstruct topologies under the Monotonic Condition.

\section{B. Synthetic Measurement Experiments}

Synthetic measurements enable us to analyze the capabilities of our methods with full ground truth and over a range of network sizes. Here we consider both synthetic topologies generated with respect to the Heuristically Optimized Topology framework [19] and real-world networks from the Internet Topology Zoo database [13]. To synthesize pairwise similarities measurements, every topology link is assigned a random similarity value, with synthesized pairwise similarities being the sum of the node similarity values (with the smallest router similarity assigned, $\delta=0.1$ ) along the shared shortest path from the root node (randomly chosen in the topology) to the two end hosts under consideration. Due to these experiments being noise-free with similarities that satisfy the $\delta$-Margin Condition, all topology reconstruction methodologies will perfectly reconstruct the topologies from the pairwise similarity measurements. The results, in terms of the number of pairwise measurements needed to reconstruct the tree topologies, are shown with respect to 100 realizations of each topology. Where, each realization consists of choosing a random leaf node to create the topology tree and randomly permuting the leaf nodes to avoid performance bias from our ordering-based techniques.

1) Heuristically Optimized Experiments: A Heuristically Optimized Topology incorporates realistic network topology characteristics that have properties that are consistent with many of those observed in the Internet, incorporating societal, engineering, and economic constraints. Topologies satisfying this framework are created using the Orbis topology generator [20] with three different sized topologies $(N=$ $\{768,1497,2261\})$. This progression in topology sizes allows us to show performance as large-scale networks grow.

TABLE II

COMPARISON OF NUMBER OF PROBES NEEDED TO ESTIMATE LOGICAL TOPOLOGY USING SYNTHETIC ORBIS TOPOLOGIES ON PRIOR METHODOLOGIES.

\begin{tabular}{|c|c|c|c|}
\hline & $\begin{array}{l}\text { Agglomerative } \\
\text { Clustering }\end{array}$ & \multicolumn{2}{|c|}{$\begin{array}{c}\text { Sequential [12] } \\
\text { Algorithm }\end{array}$} \\
\hline $\begin{array}{c}\text { Number of } \\
\text { End Hosts }(\mathrm{N})\end{array}$ & $\begin{array}{c}\text { \# Pairwise } \\
\text { Sim. Needed }\end{array}$ & $\begin{array}{c}\text { \# Pairwise } \\
\text { Sim. Needed }\end{array}$ & $\begin{array}{c}\text { Percentage of } \\
\text { Agglomerative Pairs }\end{array}$ \\
\hline 768 & 294,528 & 52,774 & $17.9 \%$ \\
\hline 1,497 & $1,119,756$ & 112,375 & $10.0 \%$ \\
\hline 2,261 & $2,554,930$ & 128,104 & $5.0 \%$ \\
\hline
\end{tabular}

In Table II, we present the number of pairwise measurements needed by the prior tomography methodologies, agglomerative clustering and Sequential. By exploiting the tree structure, the state-of-the-art Sequential method requires at most $20 \%$ of the pairwise probes the agglomerative clustering methodology requires. In Table III, we present the resulting number of pairwise similarities required to resolve the logical topology for the MONOTONICTOPOLOGY and MARGINTopologY methodologies. As seen in the tables, both DFS Ordering-based methodologies do significantly better than the exhaustive agglomerative clustering approach. In terms of the 
TABLE I

THE TREE RECONSTRUCTION METHODOLOGIES FOR A BALANCED $\ell$-ARY TREE. (WHERE $p(\ell)=\left(\frac{\ell+1}{2}-\frac{1}{\ell}\right)$ IS SUBLINEAR IN $\ell$ )

\begin{tabular}{|l|c|c|c|}
\hline Methodology & $\begin{array}{c}\text { Pairwise Probe } \\
\text { Upper Bounds }\end{array}$ & $\begin{array}{c}\text { Satisfies } \delta \text {-Margin-based } \\
\text { Reconstruction? }\end{array}$ & $\begin{array}{c}\text { Satisfies Monotonic-based } \\
\text { Reconstruction? }\end{array}$ \\
\hline \hline Sequential [12] & $\ell N \log _{\ell} N$ & Yes & No \\
MARGINTOPOLOGY & $N\left(p(\ell) \log _{\ell} N+1\right)$ & Yes & No \\
\hline \hline Agglomerative Clustering & $\frac{1}{2} N(N-1)$ & Yes & Yes \\
MONOTONICTOPOLOGY & $N\left((\ell+9) \log _{2} N+1\right)$ & Yes & Yes \\
\hline
\end{tabular}

768-end host topology, we obtain a savings with requiring less than $2 \%$ of the pairwise measurements used by the exhaustive agglomerative clustering approach. As expected, due to the more restrictive conditions, the MARGINTOPOLOGY methodology consistently requires fewer pairwise similarities than the MONOTONICTOPOLOGY methodology. The MARGINTOPOLOGY method requires at most $10 \%$ of the number of pairwise similarities required by the Sequential method. Meanwhile, even the MONOTONICTOPOLOGY methodology (which does not require the $\delta$-Margin Condition) outperforms the Sequential methodology by requiring less than $15 \%$ of the number of pairwise probes used by this state-of-the-art approach.

2) Topology Zoo Experiments: Using the Internet Topology Zoo database [13], we obtain 21 real world network topologies to observe the performance of the topology discovery algorithms. In Table IV, we find that for every network under consideration, the MARGINTOPOLOGY methodology outperforms the prior techniques (i.e., Sequential and Agglomerative Clustering). With the exception of the small Abliene topology (with only 11 routers included), the MONOTONICTOPOLOGY method outperforms the two prior techniques. This information is presented visually in Figure 5, where we show how the number of required pairwise measurements scales for each method as the size of the network increases.

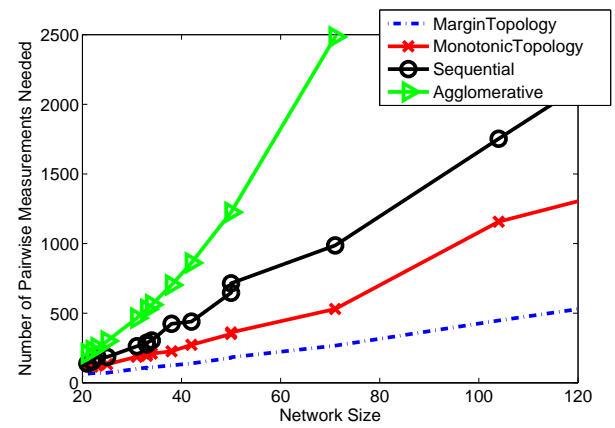

Fig. 5. Comparison of pairwise measurements needed to resolve Internet Topology Zoo networks (results presented averaged across 100 random realization of each network).

\section{Real World Measurement Experiments}

To observe the performance of our algorithm on realworld topologies, we chose 9 DNS servers located at smallto-medium sized colleges in the New England geographic area. Using the DNS server addresses and traceroute probes we discovered the following logical tree topology in
Figure 6 starting at the University of Wisconsin - Madison as the root node. While this small study focuses on end hosts all connected with Internet 2 and existing in the same general geographic area, it will address the challenges of using network tomography in the live Internet with observed measurements.

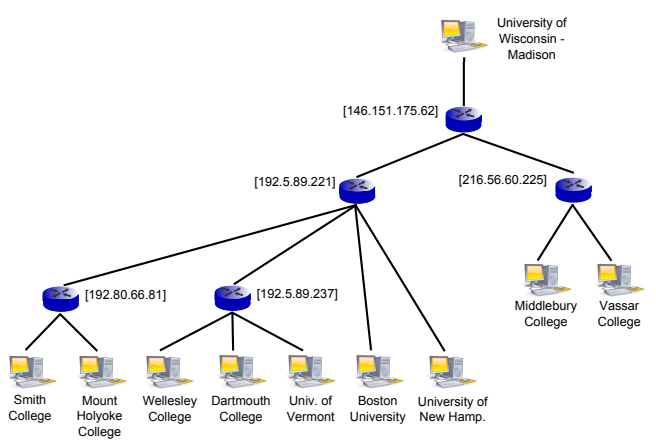

Fig. 6. Real world topology used to test tomography methods

Using delay-based unicast tomographic probes, the pairwise similarities were found between pairs of the end hosts in the topology. The delay-based unicast tomographic technique we will focus on is Network Radar [10]. Network Radar uses round trip time (RTT) measurements as the basis for topology inference and was developed as an attempt to obviate the need for significant coordinated measurement infrastructure. Consider the simple logical topology in Figure 7. Back-toback packets originating from end host $a$ will travel along the same path until router $R$. It can be assumed that any delays encountered before router $R$ induced by router queuing delays will cause highly correlated delays for both back-toback packets (due to both packets being in the same router queues). Assuming that any delays encountered between the two packets past router $R$ are uncorrelated ([10]), then the level of covariance between the RTT delays found from a series of back-to-back packets $\left(\operatorname{cov}\left(d_{b}, d_{c}\right)\right)$ will inform us to the amount of shared logical topology between paths $\{a, b\}$ and $\{a, c\}$. In order to be more robust to observed delay with high variance, for our real world experiments the pairwise similarity will be considered as the correlation coefficient of the observed pairwise delay vectors, $s_{b, c}=\frac{\operatorname{cov}\left(d_{b}, d_{c}\right)}{\sqrt{\operatorname{var}\left(d_{b}\right) \operatorname{var}\left(d_{c}\right)}}$.

Using the Network Radar methodology, each pairwise similarity is obtained using 1,500 back-to-back round-trip-time delay samples. Due to imperfect round-trip-time measurements and other delay noise measured, the delay-based similarity was found to not be perfectly correlated to the traceroute observed shared path length. As explored in [10], the number 
TABLE III

COMPARISON OF NUMBER OF PAIRWISE MEASUREMENTS NEEDED TO ESTIMATE LOGICAL TOPOLOGY USING SYNTHETIC ORBIS TOPOLOGIES (RESULTS PRESENTED AVERAGED ACROSS 100 RANDOM REALIZATION OF EACH NETWORK).

\begin{tabular}{|c|c|c|c|c|c|c|}
\hline & \multicolumn{3}{|c|}{ MARGINTOPOLOGY Algorithm } & \multicolumn{3}{|c|}{ MONOTONICTOPOLOGY Algorithm } \\
\hline End Hosts $(\mathrm{N})$ & $\begin{array}{c}\text { \# Pairwise } \\
\text { Sim. Needed }\end{array}$ & $\begin{array}{c}\text { Percentage of } \\
\text { Agglomerative Probes }\end{array}$ & $\begin{array}{c}\text { Percentage of } \\
\text { Sequential Probes }\end{array}$ & $\begin{array}{c}\text { \# Pairwise } \\
\text { Sim. Needed }\end{array}$ & $\begin{array}{c}\text { Percentage of } \\
\text { Agglomerative Probes }\end{array}$ & $\begin{array}{c}\text { Percentage of } \\
\text { Sequential Probes }\end{array}$ \\
\hline $\begin{array}{c}768 \\
1,497 \\
2,261\end{array}$ & $\begin{array}{c}3,604 \\
7,771 \\
11,848\end{array}$ & $\begin{array}{l}1.22 \% \\
0.69 \% \\
0.46 \%\end{array}$ & $\begin{array}{l}6.83 \% \\
6.92 \% \\
9.25 \%\end{array}$ & $\begin{array}{c}5,511 \\
10,571 \\
17,929\end{array}$ & $\begin{array}{l}1.87 \% \\
0.94 \% \\
0.70 \%\end{array}$ & $\begin{array}{c}10.44 \% \\
9.41 \% \\
14.0 \%\end{array}$ \\
\hline
\end{tabular}

TABLE IV

COMPARISON OF NUMBER OF PAIRWISE MEASUREMENTS NEEDED TO ESTIMATE LOGICAL TOPOLOGY USING VARIOUS INTERNET TOPOLOGY ZOO NETWORKS (RESULTS PRESENTED AVERAGED ACROSS 100 RANDOM REALIZATION OF EACH NETWORK).

\begin{tabular}{|c|c|c|c|c|c|}
\hline $\begin{array}{l}\text { Network } \\
\text { Name }\end{array}$ & $\begin{array}{l}\text { Network } \\
\text { Size }\end{array}$ & $\begin{array}{l}\text { MARGINTOPOLOGY } \\
\text { Methodology }\end{array}$ & $\begin{array}{c}\text { MONOTONICTOPOLOGY } \\
\text { Methodology }\end{array}$ & $\begin{array}{c}\text { Sequential } \\
\text { Methodology [12] }\end{array}$ & $\begin{array}{l}\text { Agglomerative } \\
\text { Clustering [14] }\end{array}$ \\
\hline Global Center & $\overline{99}$ & 17.00 & 31.59 & 35.00 & 36 \\
\hline Abliene & 11 & 25.57 & 43.75 & 41.73 & 55 \\
\hline BT (Asia) & 16 & 47.14 & 75.80 & 88.74 & 120 \\
\hline WIDE & 16 & 44.83 & 76.29 & 78.54 & 120 \\
\hline MCI & 19 & 50.91 & 93.85 & 111.88 & 171 \\
\hline Quest & 20 & 55.66 & 103.25 & 119.84 & 190 \\
\hline Vinaren & 21 & 64.25 & 119.53 & 138.11 & 210 \\
\hline BT (Europe) & 22 & 69.01 & 116.05 & 153.47 & 231 \\
\hline IIJ & 23 & 69.87 & 123.34 & 190.21 & 253 \\
\hline ATT & 25 & 72.26 & 133.17 & 185.29 & 300 \\
\hline TiNET & 31 & 99.59 & 187.24 & 262.36 & 465 \\
\hline BICS & 33 & 109.41 & 206.33 & 275.48 & 528 \\
\hline BT (North America) & 33 & 102.75 & 194.53 & 288.05 & 528 \\
\hline Geant & 34 & 111.55 & 210.12 & 303.71 & 561 \\
\hline China Telecom & 38 & 125.18 & 226.46 & 423.91 & 703 \\
\hline UUNET & 42 & 138.86 & 272.60 & 439.65 & 861 \\
\hline DFN & 50 & 178.62 & 350.62 & 646.23 & 1,225 \\
\hline Bell South & 50 & 183.38 & 361.64 & 714.58 & 1,225 \\
\hline TW & 71 & 266.48 & 530.37 & 986.39 & 2,485 \\
\hline Cogent & 104 & 447.39 & $1,157.05$ & $1,753.22$ & 5,356 \\
\hline GTS & 131 & 584.86 & $1,405.23$ & $2,373.82$ & 8,515 \\
\hline
\end{tabular}

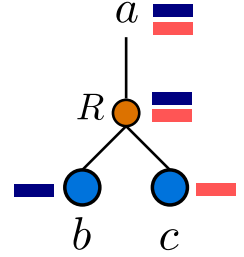

Fig. 7. Example of Network Radar on simple logical topology.

of back-to-back delay probes sent will determine the accuracy with which the observed correlation coefficient matches the underlying topology. We calculate how well our delay-based pairwise similarity matches the two specified conditions on the underlying topology, the $\delta$-Margin Condition (for three values of $\delta,=0.1,0.2,0.3)$ and Monotonic Condition, in Figure 8.

For the end hosts triple of Vassar College, Smith College, and Boston University in Figure 8-(Left), we find that very few delay measurements are needed such that the calculated pairwise similarities satisfy the Monotonic Condition with respect to the true underlying topology for this triple, but significantly more delay probes are needed to satisfy the restrictive $\delta$ Margin Condition. For the end hosts triple of Vassar College, Smith College, and Dartmouth College in Figure 8-(Right), we again find that the less restrictive Monotonic Condition is satisfied with few delay measurements, but for the three values of $\delta$ under consideration, the $\delta$-Margin Condition is rarely satisfied. This is due to the $\delta$-Margin Condition requiring both a similarity separation of at least $\delta$ between pairs of similarities corresponding to different shared path lengths, and requiring strictly less than $\delta$ similarity separation between pairs of similarities corresponding to equal shared path lengths. When either of these properties are violated, then the $\delta$-Margin Condition will not hold. In contrast, the Monotonic Condition only requires that pairwise similarities corresponding to longer shared path lengths have values greater than pairwise similarities corresponding to shorter shared path lengths, a much less restrictive condition and therefore satisfied with fewer delay probes.

For any estimation procedure based on Internet measurements, there will be the potential for errors in the reconstructed topology ${ }^{1}$. In order to determine the accuracy of our estimated topologies, we must develop a metric that compares our estimated topologies to the ground-truth topology. We consider the following accuracy measure for our reconstructed tree topologies. Given a triple of end hosts $\{a, b, c\}$ that exists in our estimated topology, we can predict whether there is a longer shared path between end hosts $\{a, b\}$ or end hosts $\{a, c\}$. For the estimated logical topology $\widehat{\mathcal{T}}$, these two paths will be denoted $\widehat{p}_{a, b}$ and $\widehat{p}_{a, c}$ respectively. And

\footnotetext{
${ }^{1}$ This could be improved upon by taking more back-to-back sample probes or using a DAG card to obtain more accurate time information, but for this paper we will focus on the case where neither improvement is available.
} 

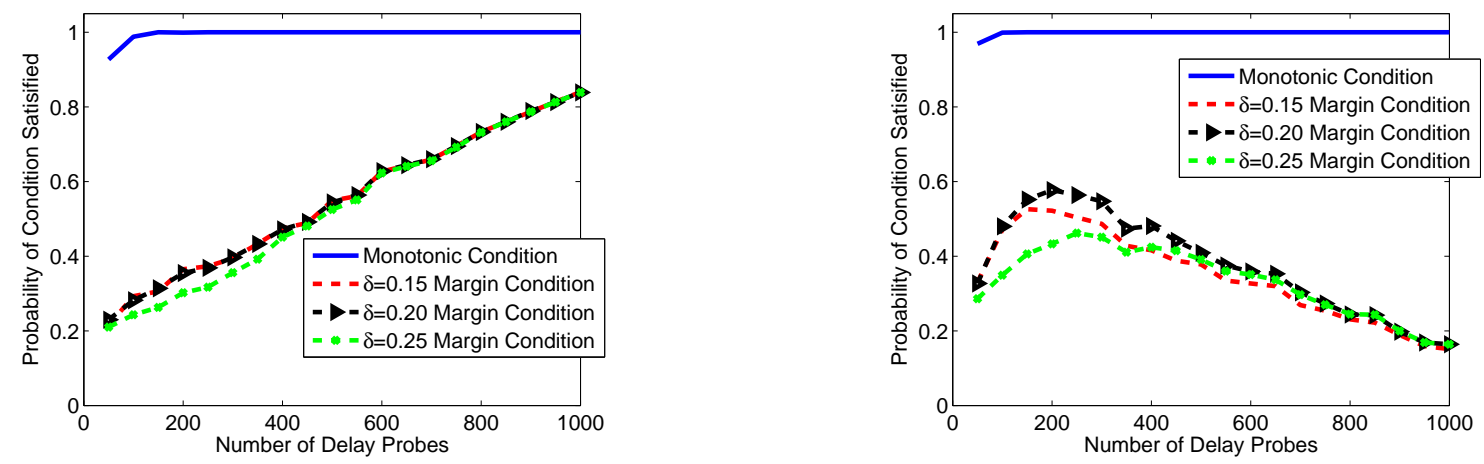

Fig. 8. For a specified triple of end hosts, the probability of the calculated pairwise similarities satisfying the Monotonic and $\delta$-Margin Conditions (with results averaged across 1,000 random selections from 1,500 total delay measurements). (Left) - Vassar College, Smith College, and Boston University hosts, and (Right) - Vassar College, Smith College, and Dartmouth College hosts.

for the true topology, these two true path lengths will be denoted as $p_{a, b}$ and $p_{a, c}$ respectively. The more accurate our estimated topology, the more often our estimated topology will return the correct answer for whether $\{a, b\}$ has more shared infrastructure than $\{a, c\}$. The percentage of times we are correct with this problem will be denoted as $p$. For all possible triples in our set of end hosts $(\mathbf{X})$, this shared path classification rate can be found by,

$$
p=f(\mathbf{X}) \sum_{a \in \mathbf{X}} \sum_{b \in \mathbf{X}} \sum_{c \in \mathbf{X}} \mathbf{1}\left(\widehat{p}_{a, b}>\widehat{p}_{a, c}\right) \mathbf{1}\left(p_{a, b}>p_{a, c}\right)
$$

With the value,

$$
f(\mathbf{X})=\left(\sum_{a \in \mathbf{X}} \sum_{b \in \mathbf{X}} \sum_{c \in \mathbf{X}} \mathbf{1}\left(p_{a, b}>p_{a, c}\right)\right)^{-1},
$$

Where $\mathbf{1}(x)=1$ if the condition $x$ holds while $\mathbf{1}(x)=0$ if the condition $x$ does not hold.

The baseline for any topology reconstruction algorithm will be to outperform a naive randomly reconstructed topology with end hosts and interior nodes connected at random into a tree topology. Our shared path classification rate will be the metric we use to assess the accuracy of our estimated topologies.

Due to the Sequential Algorithm and both DFS Ordering algorithms having performance sensitive to initial ordering of end hosts, the performance of the three algorithms are averaged over 500 random permutations of the end hosts. Averaging over many random permutations eliminates any order bias from the results.

The two margin-based methodologies (Sequential and MARGINTOPOLOGY) have a tunable margin parameter, $\delta$, that must be chosen. To give the prior margin-based methodology every possible advantage, for each experiment the performance of the Sequential algorithm is shown for the best possible value of $\delta$ at each level of probing. Meanwhile, our new MARGINTOPOLOGY methodology has a constant value of $\delta$ across all levels of probing.

For the real-world topology in Figure 6, the corresponding shared path classification rate (from Equation 2) for the $\delta$ Margin Condition algorithms (i.e., MARGINTOPOLOGY and Sequential) and a baseline random methodology can be seen in
Figure 9-(Left) versus a restricted total number of delay probes available. We find that the MARGINTOPOLOGY methodology performs significantly better than both the Sequential and random topologies. Surprisingly, the Sequential methodology requires a large number of pairwise probes to outperform the random topologies, we believe this is due to the need for a margin $\delta$ between the similarity measurements with different shared path values. While this methodology also requires the $\delta$-Margin Condition, through the exploitation of DFS ordering, our methodology will be more robust to violations of the margin condition (as evident by the improved performance). Given the probing complexity in Table I, it is very likely that the accuracy improvements for the DFS Ordering-based techniques will further grow as the size of the topology increases.

The shared path classification rate (from Equation 2) for the two monotonic-based topology reconstruction algorithms (i.e., MONOTONICTOPOLOGY and agglomerative clustering) can be seen in Figure 9-(Right) versus a restricted total number of delay probes available. As seen in the figure, for a wide range of available pairwise probes, the MONOTONICTOPOLOGY methodology more accurately resolves the tree topology than the standard bottom-up agglomerative clustering methodology. For example, to obtain the same tree reconstruction accuracy $(p=0.7)$, the MONOTONICTOPOLOGY methodology requires 1,700 fewer delay-based measurements sent through the network compared with the exhaustive agglomerative clustering methodology $(3,800$ delay probes for MONOTONICTOPOLOGY, while agglomerative clustering requires 5,600 delay probes).

\section{CONCLUSions / Future Work}

Despite concerted efforts, generating accurate maps of the router-level topology of the Internet remains a compelling objective in Internet measurement. Standard TTL-based and Record Route methods for discovering router-level network topology have well known limitations that motivate development of alternative topology measurement methods. One such method is the application of tomographic inference to recover the underlying topology. While network tomography 

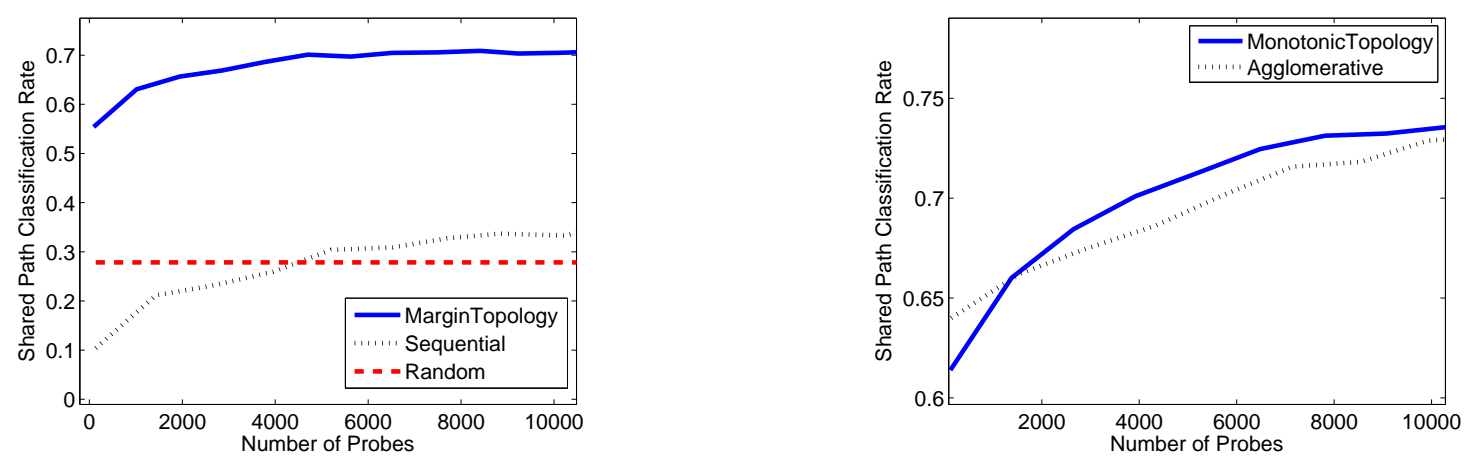

Fig. 9. (Left) - Topology reconstruction results for the two $\delta$-Margin Condition algorithms (MARGINTopology, Sequential) and a baseline random topology. (Right) - Topology reconstruction results for the two Monotonic Condition algorithms (MONOTONICTOPOLOGY and agglomerative clustering).

for topology discovery has been examined in the past, it is yet to be widely used in practice due to its own set of limitations.

The goal of our work is to address the shortcomings of measurement-based network tomography for discovering Internet logical topology. Tomographic methodologies described in prior work required an impractical number of probes. In this paper, we describe algorithms that considerably reduce the number of pairwise probes needed to resolve logical topologies. The ability to reduce the number of pairwise probes is reliant on exploiting the idea of a Depth-First Search (DFS) Ordering of the end hosts. We analyze the capabilities of our algorithms on a set of large-scale synthetically generated topologies. The experiments on these topologies show our new methodologies require only $2 \%$ of the probes used by an exhaustive methodology, and roughly $15 \%$ of the probes used by the current state-of-the-art. Results from a small-scale realworld Internet experiment further validate the performance of our algorithms. The significant reduction in the number of probes needed opens this efficient discovery technique to new avenues of applications, including general clustering problems using pairwise similarities. In addition, future work will look to extend these techniques from reconstructing tree topologies to reconstructing graph topologies.

\section{APPENDIX}

\section{A. Proof of Proposition 3}

Using Algorithm 2, consider the first step, end host $x_{1}$ will be chosen and the similarity values will be found between $x_{1}$ and $x_{2}, x_{3}, \ldots, x_{N}$. Given the $\ell$-ary balanced property of the tree and the $\delta$-margin condition, after sorting the similarity values this implies that the first iteration of the algorithm will divide the set of end hosts into a group of $\frac{N}{\ell}$ end hosts and a group of $\frac{(\ell-1) N}{\ell}$ end hosts corresponding to the first branch on the first level of the tree as seen in Figure 10-(Left). Consider further subdividing the set of $\frac{(\ell-1) N}{\ell}$ end hosts, where a random end host is chosen in the set and $\frac{(\ell-1) N}{\ell}-1$ similarity measurements are taken. Our bisection algorithm would then subpartition into a group of $\frac{N}{\ell}$ end hosts and a group of $\frac{(\ell-2) N}{\ell}$ end hosts, again corresponding to the first level of the tree as seen in Figure 10-(Right). In these initial steps of the algorithm each iteration is resolving a branch off the first level of this tree, clustering into $\ell$ sets of $\frac{N}{\ell}$ end hosts each relating to a branch off the first level of the tree.
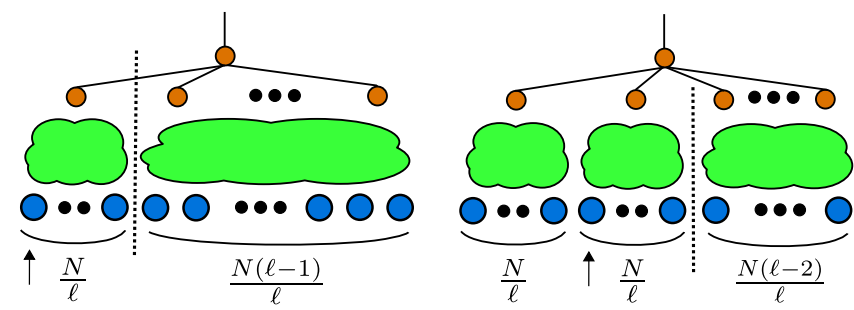

Fig. 10. (Left) The first split taken on a balanced $\ell$-ary tree. (Right) The second split taken on a balanced $\ell$-ary tree. Both splits indicated by the dotted line, the arrow indicates the randomly chosen end host similarity values are measured against.

After the tree has been divided past the first level, the problem can now be considered ordering the $\ell$ number of subtrees each with $\frac{N}{\ell}$ end hosts. Using this recursive property, we can state the number of probes needed for a balanced $\ell$-ary tree with $N$ leaf nodes as $f_{\ell}(N)$.

$$
\begin{aligned}
f_{\ell}(N) & \leq N+\frac{\ell-1}{\ell} N+\cdots+\frac{2}{\ell} N+\ell f_{\ell}\left(\frac{N}{\ell}\right) \\
& =\frac{N}{\ell}(2+3+\cdots+\ell)+\ell f_{\ell}\left(\frac{N}{\ell}\right) \\
& \stackrel{(a)}{=} N p(\ell)+\ell f_{\ell}\left(\frac{N}{\ell}\right) \\
& \stackrel{(b)}{\leq} N p(\ell)+\ell\left\{\frac{N}{\ell} p(\ell)+\ell f_{\ell}\left(\frac{N}{\ell^{2}}\right)\right\} \\
& =2 N p(\ell)+\ell^{2} f_{\ell}\left(\frac{N}{\ell^{2}}\right) \\
& \vdots \\
& \stackrel{(c)}{\leq} \quad p(\ell) N\left(\log _{\ell} N\right)
\end{aligned}
$$

Where

$$
p(\ell):=\frac{(2+3+\cdots+\ell)}{\ell}=\left(\frac{\ell+1}{2}-\frac{1}{\ell}\right)
$$

\section{B. Proof of Proposition 3}

Consider a balanced $\ell$-ary tree with a set of $\mathrm{N}$ end hosts $X=\left\{x_{1}, x_{2}, \ldots, x_{N}\right\}$. Let $f_{\ell}(N)$ denote the number of 
pairwise measurements required to discover a DFS ordering on the set $X$ and let $s_{m}(N) \leq m^{2} \log _{m} N$ be the number of measurements required to find the exact split by agglomerative clustering $m$ chosen end hosts out of $N$ and proceeding recursively (as indicated by Algorithm 4) until the split point $x^{*}$ is found. Using the recursive property of the algorithm, we can state:

$$
\begin{aligned}
& f_{\ell}(N) \leq\left(N+s_{m}(N)\right)+ \\
& \left(\frac{\ell-1}{\ell} N+s_{m}\left(\frac{\ell-1}{\ell} N\right)\right)+\cdots \\
& \cdots+\left(\frac{2}{\ell} N+s_{m}\left(\frac{2}{\ell} N\right)\right)+\ell f_{\ell}\left(\frac{N}{\ell}\right) \\
& \stackrel{(a)}{=} N p(\ell)+\sum_{i=2}^{\ell} s_{m}\left(\frac{i}{\ell} N\right)+\ell f_{\ell}\left(\frac{N}{\ell}\right) \\
& \stackrel{(b)}{\leq} N p(\ell)+\ell m^{2} \log _{m} N+\ell f_{\ell}\left(\frac{N}{\ell}\right) \\
& \leq N p(\ell)+\ell m^{2} \log _{m} N+ \\
& \ell\left\{\frac{N}{\ell} p(\ell)+\ell m^{2} \log _{m}\left(\frac{N}{\ell}\right)+\ell f_{\ell}\left(\frac{N}{\ell^{2}}\right)\right\} \\
& \leq 2 N p(\ell)+\left(\ell+\ell^{2}\right) m^{2} \log _{m} N+\ell^{2} f_{\ell}\left(\frac{N}{\ell^{2}}\right) \\
& \leq N p(\ell) \log _{\ell} N+\left(\sum_{j=1}^{\log _{\ell} N} \ell^{j}\right) m^{2} \log _{m} N \\
& \leq N p(\ell) \log _{\ell} N+\frac{N-1}{\ell-1} m^{2} \log _{m} N \\
& \leq N \ell \log _{\ell} N+N m^{2} \log _{m} N
\end{aligned}
$$

In $(a), p(\ell):=\frac{2+3+\cdots+\ell}{\ell}=\frac{\ell+1}{2}-\frac{1}{\ell}$.

Given that we control the agglomerative clustering procedure in Algorithm 5, we can reduce the total pairwise measurements needed by setting $m=3$ (as we require $m \geq$ 3 ). This results in the total pairwise measurements needed by Algorithm 5 to resolve a DFS ordering to be less than $N(\ell+9) \log _{2} N$ measurements for $N$ end hosts clustered in a $\ell$-ary balanced tree. Combined with Theorem 1 , we see that to resolve the tree topology requires only $N\left((\ell+9) \log _{2} N+1\right)$ pairwise similarities that satisfy the monotonic condition.

\section{REFERENCES}

[1] B. Donnet, P. Raoult, T. Friedman, and M. Crovella, "Deployment of an Algorithm for Large-Scale Topology Discovery," in IEEE Journal of Selected Areas in Communications, Special Issue on Sampling the Internet, vol. 24, 2006, pp. 2210-2220.

[2] N. Spring, R. Mahajan, and D. Wetherall, "Measuring ISP Topologies with Rocketfuel," in Proceedings of ACM SIGCOMM, Pittsburgh, PA, August 2002.

[3] B. Yao, R. Viswanathan, F. Chang, and D. Waddington, "Topology Inference in the Presence of Anonymous Routers," in Proceedings of IEEE INFOCOM, San Francisco, CA, 2003, pp. 353-363.

[4] M. H. Gunes and K. Sarac, "Resolving ip aliases in building traceroutebased internet maps," IEEE/ACM Transactions on Networking, vol. 17, pp. 1738-1751, December 2009.

[5] R. Sherwood and N. Spring, "Touring the Internet in a TCP Sidecar," in Proceedings of ACM SIGCOMM Internet Measurements Conference, Rio de Janeriro, Brazil, 2006, pp. 339-344.

[6] R. Sherwood, A. Bender, and N. Spring, "DisCarte: A Disjunctive Internet Cartographer," in Proceedings of ACM SIGCOMM, Seattle, WA, August 2008.
[7] N. Duffield and F. L. Presti, "Network Tomography from Measured Endto-End Delay Covariance," in IEEE/ACM Transactions on Networking, vol. 12 , no. 6, 2004, pp. $978-992$.

[8] N. Duffield, J. Horowitz, and F. L. Presti, "Adaptive Multicast Topology Inference," in Proceedings of IEEE INFOCOM, Anchorage, AK, 2001, pp. 1636-1645.

[9] R. C. M. Coates and R. Nowak, "Maximum Likelihood Network Topology Identification from Edge-Based Unicast Measurements," in Proceedings of ACM SIGMETRICS, Marina Del Rey, CA, June 2002.

[10] Y. Tsang, M. Yildiz, P. Barford, and R. Nowak, "Network radar: tomography from round trip time measurements," in Proceedings of ACM SIGCOMM Internet Measurements Conference, Taormina, Sicily, Italy, 2004, pp. 175-180.

[11] N. Duffield, F. L. Presti, V. Paxson, and D. Towsley, "Network Loss Tomography using Striped Unicast Probes," in IEEE/ACM Transactions on Networking, vol. 14, no. 4, 2006, pp. 697-710.

[12] J. Ni, H. Xie, S. Tatikonda, and Y. R. Yang, "Efficient and dynamic routing topology inference from end-to-end measurements," in IEEE/ACM Transactions on Networking, vol. 18, no. 1, February 2010, pp. 123-135.

[13] S. Knight, H. X. Nguyen, N. Falkner, R. Bowden, and M. Roughan, "The Internet Topology Zoo." [Online]. Available: http://www.topology-zoo. org

[14] T. Hastie, R. Tibshirani, and J. Friedman, The Elements of Statistical Learning. Springer, 2001.

[15] R. Castro, M. Coates, and R. Nowak, "Likelihood Based Hierarchical Clustering," in IEEE Transactions on Signal Processing, vol. 52, August 2004, pp. 2308-2321.

[16] A. B. Kahn, "Topological Sorting of Large Networks," in Communications of the ACM, vol. 5, 1962, pp. 558-562.

[17] M. Qiu, C. Xue, Z. Shao, Q. Zhuge, M. Liu, and E. Sha, "Efficent Algorithm of Energy Minimization for Heterogeneous Wireless Sensor Network," in Embedded and Ubiquitous Computing, Lecture Notes in Computer Science, 2006, pp. 25-34.

[18] B. Eriksson, G. Dasarathy, P. Barford, and R. Nowak, "Toward the practical use of network tomography for internet topology discovery," in Proceedings of IEEE INFOCOM, San Diego, CA, March 2010.

[19] D. Alderson, L. Li, W. Willinger, and J. Doyle, "Understanding Internet Topology: Principles, Models and Validation," IEEE/ACM Transactions on Networking, vol. 13, no. 6, pp. 1205-1218, December 2005.

[20] P. Madadevan, C. Hubble, D. Krioukov, B. Huffaker, and A. Vahdat, "Orbis: Rescaling Degree Correlations to Generate Annotated Internet Topologies," in Proceedings of ACM SIGCOMM, Kyoto, Japan, August 2007. 